\title{
A MULTIWIRE PROPORTIONAL CHAMBER FOR IMAGING THERMAL, EPICADMIUM, AND FAST NEUTRONS
}

Kenneth Valentine and Selig Kaplan

Law rence Berkeley Laboratory, Bcrkeley and

Department of Nuclear Engineering.

University of California. Berkeley

Victor Perez-Mendez

Lawrence Berkeley Laboratory. Berkeley ard Department of Radiology

University of California, San Francisco

$$
\text { Leon Kaufman }{ }^{*}
$$

Department of Radiology

University of California, San Francisco

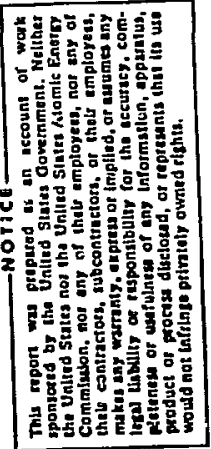

\section{Abstract}

The $25 \mathrm{~cm} \times 25 \mathrm{~cm}$ chamber described images hermal and epicadmium neutron beams using two loron-coated conversio's screens, and fast reutrons ssing a single polyethylene converter. Sample images ind quantitative attenuation and resolution data are rresented. Measurements of neutron attenuation in ucite patterns give good agreement with expected ralues for thermal, epicadmium, and fast (Pu-Be) reams. The measured mean free paths were 0.30 , J.81. and $5.8 \mathrm{~cm}$. respectively. Thermal neutron resolution $(0.2 \mathrm{~cm})$ depends upon a-particle range, sut resolution for fast neutrons $(0.4 \mathrm{~cm})$ appears to se limited by chamber thickness (to approximately jne-half of the chamber thickness). Measurements involving digital image storage and manipulation are ulso described and illustrated.

\section{Introduction}

Multiwire proportional chambers with delayline readouts have been demonstrated to have the capability for moderate resolution, mqderate cost. high sensitivity radiographic imaging. 1. Important features of such detactor systems are the ability to image single interactions, and the ability to provide position information over a large sensitive area in a form that is easily digitized for computer storage and manipulation. In as earlier paper ${ }^{3}$ calculations were made of the performance of such a chamber in conjunction with a ${ }^{10} 0_{B}$ converter, as a thermal-neutron, radiographic imaging device. (Some simple pattern images were produced with a small prototype, $4 \mathrm{~cm} \times 4.5 \mathrm{~cm}$, chamber). This work has now been exterided through the constructiun of a larger. more efficient syotem that has been used for fastneutron as well as the rmal-neutron imaging.

\footnotetext{
Work dane under the auspices of the United States Atomic Energy Commission.
}

'5upported by U.S. Public Health Service Research Career Award 70598-02 from the NIGMS.

\section{Chamber Construction}

An exploded drawing of the cliamber is shown in Fig. 1. The chamber has an active area of $25 \mathrm{~cm}$ $\times 25 \mathrm{~cm}$. The central anode plane is a parallel array of 12.5-um-diameter gold-plated tungsten wires with a spacing of $1.5 \mathrm{~mm}$ and electrically terminated at both ends to a common bus. The outer eathode grids consist of $37-\mu m-d i a m e t e r$ wires with a spacing of $1.0 \mathrm{~mm}$. The dielectric frames which separate the wire planes are made from Nemn G-10 glass epoxy. In all there

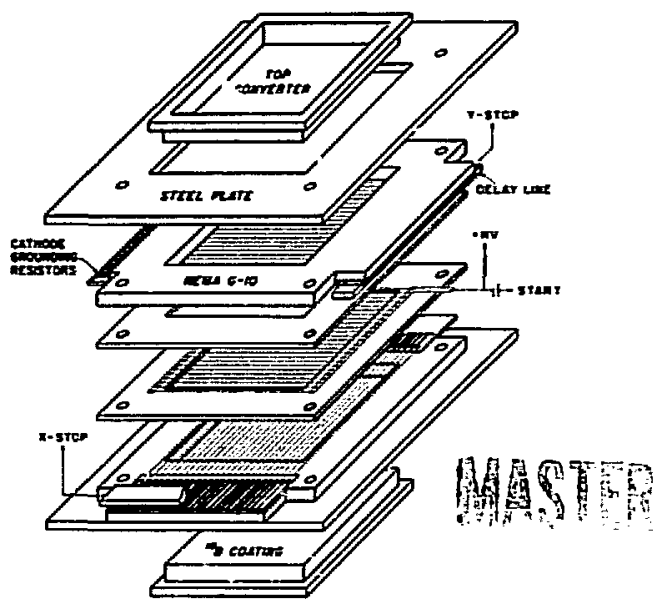

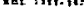

Fig. 1. Exploded view of wire chamber and delay lines. Part of the $x$-coordinate delay line is left out to show the signal coupling etrips more effectively. 
are four such frames. The two central frames are each $3 \mathrm{~mm}$ thick and provide the actuai inter-planar spacing while the outer frames are each $10 \mathrm{~mm}$ thick and provide structural rigidity during assembly. Outside frames also serve as mounting surfaces for the coupling-strip PC boards and the cGisierter plates. The four-frame subassembly is sandwiched between two 6-mm-thick steel plates and four corner bolts clamp the unit together. The frames are all surface ground to insure uniform plane spacing. The outer flange of the interchangeable aluminum converter substrate trakes an air-tight seal on the outer epoxy frame while positioning the converter surface $0.5 \mathrm{~mm}$ from the cathode wire plane. This converter plate te mechanically anchored and electrically grounded to the outer steel plate.

The anode plane is blased typically at $+2000 \mathrm{~V}$ and provides the "stirt" signal for two time-toheight converters. The two cathode-grid planes have theis wire axes mutually orthogonal. At one edge of the cathode plane the wires are terminated in pairs through a $200 \mathrm{k} \Omega$ resistor to a common bus. At the other end each is terminated separately to a flat strip on the circuit board. A delay line ${ }^{\text {is }}$ mounted in the space under the steel plase and capacitively coupled to these strips through a $0.5-\mathrm{mil}$ Mylar sheet. A pressure contact to the printed circult board is maintained by foam-rubber strips compressed into the space between the delay line and the steel plaze. The delay lines are $30 \mathrm{~cm}$ long, have a delay of $6.8 \mathrm{ntec} / \mathrm{mm}$, and give a signal attenuation of about $0.1 \mathrm{db} / \mathrm{cm}$. The common bus of the cathode planes can be positively biased to provide a drift field between the converter plane and the cathode. Pulses obtained from the delay lines are used to stop the time-to-height converters whose output-pulse amplitudes are then proportional to the $x-y$ coordinates of the event.

\section{Neutron Converters}

The neutron converting material was fixed to the Inside surfaces of the converter substrates. For thermal and epicadmium neutron detection, 92 \% enriched $10_{B}$ was used as the converting matprial. The upstream boron surface was an evaporated :oating. For the downs-ream surface, since neither total attenuation nor uniformity are considerations, a particularly aimple and equally satisfactory converter surface was made by simply dusting boron powder onto double-sided pressure-sensitive tape that was attached to the aluminum plate. The calculated rinaracteristics of such a converter surface in a proportional chamber have been described previously. 3

For fast-neutron detection a recoil-proton converter (polyethylene) is attached to the upstream converter plate. (Becauge n-p scattering all occurs in the forward hemisphere, only one converter plate can be used). Detection requires that protons scarter from the hydrogenous material into the activn volume of the chamber. A computer program was developed to model the $n-p$ collision process in a thin polyethylene converter and the subsequent escape and detection of the recoil proton.

The program determines the track end point of a recoil proton as a function of three oarameters: incident neutron energy, scattering angie, and the depth in the converter at which the scattering interaction occurs. The point at which the chamber will localize the event is taken as the center of ioniza. tion in the gas volume. By performing an appropriately weighted sum over the above three parameters, the spatial distribution of proton localization points due to a narrow, parallel beam of neutrons is determined. Efficiency and resolution characteristics are then extracted from this distribution. There are also provisions for setting upper and lower thresholds for energy deposition in the chamber as well as for taking account of finite chamber thickness. The principal difference between this analysis method and that for $a^{\prime} s$ from a boron converter ${ }^{3}$ is that the initial recoil proton energy is a function both of incident neutron energy and of scattering angle. whereas the initial a-particle energy is a constant depending only on the $Q$-value of the exothermic $(n, a)$ reaction.

Each curve in Fig. 2 represents the locus of residual range in proportional-chamber gas of a proton scattered at a particular depth in the converter. That is, a line from the coordinate origin to its intersection with a curve is the residual range of a proton produced at that particular depth and ocattered in that particular direction. Two families of such curves a re shown, corresponding to (a) $1-\mathrm{MeV}$ and (b) $4-\mathrm{MeV}$ neutrons normally incident on the converter. In each case, a converter thickness equal to the maximum scattered proton range 5 has been segmented into 25 layers. The numbers on alternate curves give the percent of the proton yield corresponding to a particular curve.

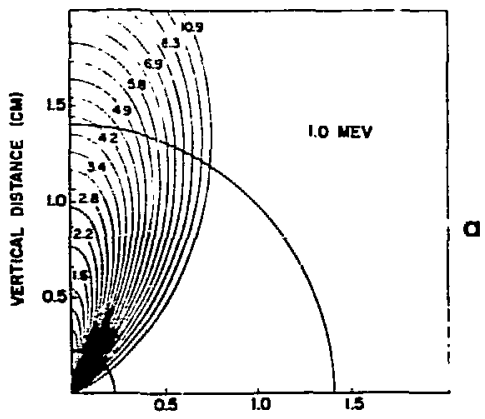

HORZONTAL DISTANCE (CW)

XBL 7310.1386

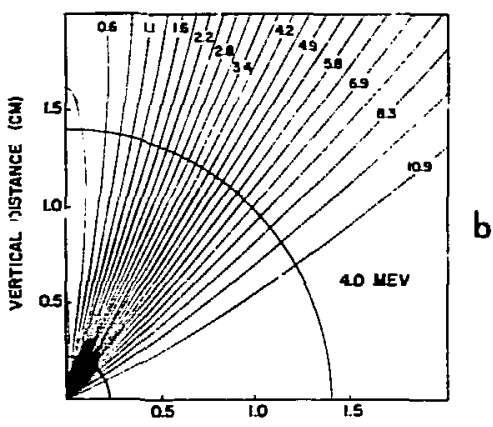

MORIOKTAL DISTAMCE (CH)

XBL 7310.1387

Fig. 2. Locus plots showing racoil proton track endings for neutron beam energies of 1.0 and $4.0 \mathrm{MeV}$. Each locus corresponds to proton emission from a different depth in the converter. 
For reference, two concentric circular segments are drawn whose radii are equal to the ranges of 0.2- and 0.7-MeV protons in argon. When the entire proton energy is deposited in the gas (i.e.. the vertical range of the proton is less than the chamber thickness) these circular curves correspond to energy discrimination levels which can be set on single channel analyzers.

Comparison of Fig. $2 a$ with $2 b$ shows that the proton ranges increase rapidly with incressing neutron energy. Fortunately the finite thickness of a practical chamber effectively terminates the proton tracks and leads to a resolution on the order of half the chamber thickness. However, the ability to select the higherenergy forward-scattered protons with energy thres holds is lost. Flgure 3 shows the calculated efficiency of a plane polyetkylene converter screen as a function of incident neutron e.ergy. Curves are shown for the actual chamber for various minimum-energydeposition thresholds. Also shown ere the response characteristics of a chamber with an infinitely thick gas volume. for example a highly pressurized chamber.

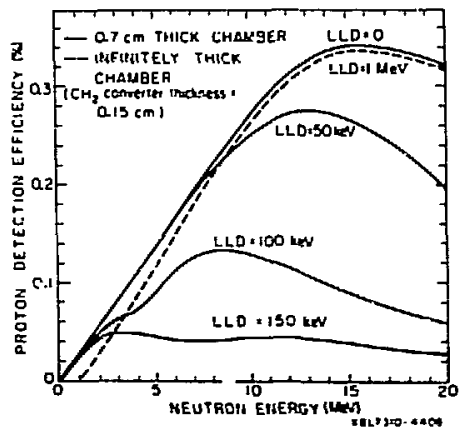

Fig. 3. Proton detection eficiency from a polyethylene recoil-proton converter vs. incident neutron energy. LLD refers to a lower level detection threahold below which protons are not included. The effect of finite chamber thickness on shifting the proton energy spectrum toward lower energies is evident.

\section{Chamber Performance}

The "intrinsic" spatial resolution if the chamber and electronics was measured by replacing one converter plate with a thin Mylar window and determining the Modulation Trangfer Function (MTF) using the 5.9-keV $x$-ray from an 55 Fe source. The MTF was compuled by Fourier transformation of the Line Spread Function (LSF) obtained from measurements with a plit collimator and correced for finite slit width. 6,7 The results of this reasuremen: are shown in Fig. 4. The MTF for the boron-plate system used as a thermal-neutron detector was then

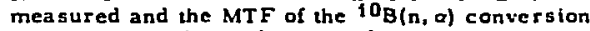
was determined as a function of spatial frequency (v) by the relationship

$$
M T F_{n, a}(v)=\frac{M T F_{\text {total }}(v)}{M T F_{\text {intrinsic }}(\nu)} .
$$

A comparison of this measured MTF and one calculated from estimated experimental parameters by the computer program is also shown in Fig. 4.

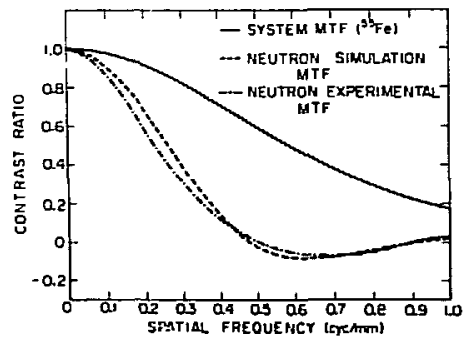

ransigenos

Fig. 4. Experimental and calculated contrast ratiog vo, spatial frequency (MTF). The thermal ne utron simulation MTF is based on estimates of the experimental parameters.

For the fast-neutron-detecting system, resolution was measured using a $\mathrm{Pu}-\mathrm{Be}$ source and a thick polyethylene "knife edgc." The 10-90\% resolution was found to be about $\$ .0 \mathrm{~mm}$ after correction for source size. The 10-90\% resolution calculated by the computer program described above was $3.5 \mathrm{~mm}$.

The maximum experimental efficiency achieved for thermal neutrons has been consistently slightly less than half of that expected from calculation. Table 1 show a comparison berween measured and calculared devetion efficiencies. The two-converter efficiency that has been achieved is in good agreement with the sum of the separately measured ef ficiencies after correction is made for beam attenuation in the first screen.

TABLE 1

Calculated and $M f^{2 s u r e d ~ E}$ Efficiencies for 92\%: 10 Converter:

\begin{tabular}{c|c|c|c}
\hline \multirow{2}{*}{$\begin{array}{c}\text { Converter } \\
\text { Thicknes: }\end{array}$} & \multicolumn{3}{|c}{ Effictency $\left(F_{0}\right)$} \\
\cline { 2 - 4 }$\left(\mathrm{mg} / \mathrm{cm}^{2}\right)$ & \multicolumn{2}{|c|}{ Calculated } & Measured \\
\hline 0 (Powder) & 5.07 & 4.09 & Maximum \\
0.86 & 4.95 & 4.09 & 2.06 \\
0.24 & 2.17 & 2.10 & 1.77 \\
& & & 1.00 \\
\hline
\end{tabular}

The calculated and measured ma*imum detec tion efficiencies for the $0.1+6 \mathrm{~g} / \mathrm{cm}^{2}=\mathrm{H}_{2}$ converier were in excellent agreement, both giving $0.10 \%$ for a Pu-Be spectrum. 8

Epicsdmium neutron radiography was explored briefly by placing a sheet of $0.020-i n$. cadmium over the face of the chamber to filter out neutrous with energies less than about $0.3 \mathrm{eV}$. Using the proportional chambe : in this mode of operation the neutrondetection ral in our reaclor beam was reduecd by 


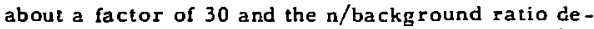
creased to 1.0. In spite of this low ratio epicadmium-image data can still be obtained because the digital nature of the chamber allons for storage and background subtraction.

\section{Some Results of Chamber Measurements}

Sample thermal-r-eutron images of an electric drill, taken with the chamber are shown in Fig. 5. The first three images show the effect of different image statistics. The fourth image was made with a 3-mm cell, gadolinium-coated, h-neycomb, antiscatter grid placed over the char iser. The pattern superimposed on the drill image, therefore, is the image of this $3-\mathrm{mm}$-cell grid. The above pictures were cbtained by using the time-to-height-converter signals to produce analogue deflections on the face of an oscillos cope. One intensified scope dot was obtained for each event and the dots were recorded with a Polaroid camera.
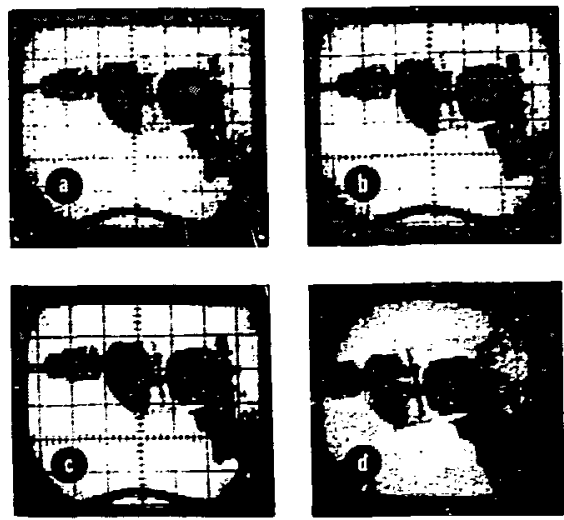

Fig. 5. Thermal neutron radiographs of an electric hand drill. Promircent features include grease in the gear case, steel armature, graphite brushes, plastic trigger. and rubber power cord. (a), (b) and (c) were taken at average neutron interaction densities of 5,10 , and $60 \mathrm{~mm}^{-2}$ respectively. (d) containg approximately 40 interactions/mm?. The experimental MTF's of Eig. 4 apply to the se radiographs.

Some preliminary work has also been done on digital storage and manipulation of chamber daci. For this work we had available only a 4096 -channel pulseheight analyzer with capabilities for two-dimensional analysis. Digital neutron-image data were obtained in a $128 \times 128$ matrix by biasing the time-to-height signals and collecting the data in four single-quadrant runs. This type of data collection, while neither ef ficient nor practical for actual eperasion. served to demonotrate the capability of the wire chamber system for such digital imaging applications. An advantage of this lype of image storage lies in the ability to normalize out local variatione in the beam intensity or the detector sensitivity. This is achieved by storing the system response to the beam alone and performing channel-by-channel division on the image matrix. Areas of the image matrix which corres pond to constant beam attenuation then contain approximately the same number (although the image statistics can vary). Digital images taken in this marner are shown in Fig. 6.

A nother example of digical imaging using pulse-heignt-analyzer-stored data is shown in Fig. $T$.
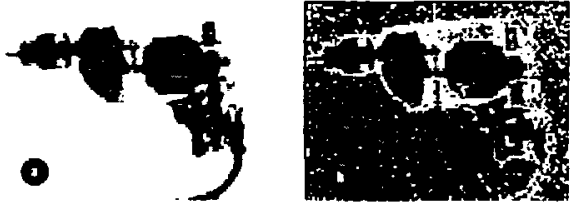

Fig. 6. Radiographs of drill collected a image matrices with PHA. Each matrix element corresponds to an image area approximately $2 \mathrm{~mm}$ square. After normalizing the unattenuated background to 1.0 as described in text, all image data lies between 0 and 1.0. In (a), 32 gray shades were evenly distributed between 0 and 1.0. In (b), 32 shades were distributed betweer 0.1 and 0.9 and data lying outside this range was imaged as black.
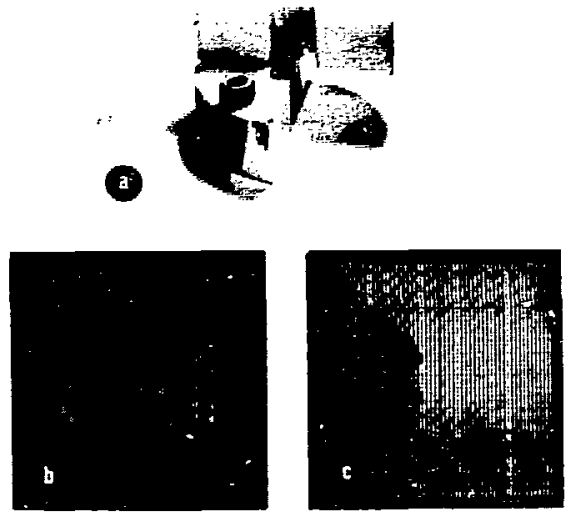

Fig. 7. a) Photograph of test object. The $3 / 4$ in. stec: nut is $i-1 / 8$ in. across the hats.

b) Neutron radiograph of test object using a Pu-Be neutron source and a Criz neutron converter. The image was stored as a $64 \times 64$ matrix in a 4096 channel analyzer. Readout was accomplished by incrementing the contour level between 64 successive core scans so the intensity of the dot representing a channel is proportional to the numerical contents of thre channel.

c) Same as (b) except the contour level was held at a constant value in order to image the stcel nus more clearly. 
This was simply a pattern made from two-in. thick pieces of polyethylene and a steel sut. This pattern was detected and imaged using the Pu-Be source. By adjusting contour thresholds as the core is scanned during CRT display, various features of the pattern can be intensified.

Digital images of various thicknesses of lucite were obtained to measure the effective thermal and epicadmium attenuation characteristics of this material for the reactor neutron spectrum. The results of these measurements together with results from $z$ Pu-Be source, with the chamber used ad a fastneutron detector, are shown in Fig. 8. For such quantitative measurements we correct for any ronuniformity in the beam or in the chamber response by using the method previously mentioned in connection with an image matrix. In all three cases the results could be fitted with a single exponential function of the form,

$$
y=C e^{-x / \lambda}+(1-C)
$$

which allows for a constant background. The values of $\lambda$ for thermal, epicadmium and fast (Pu-Be) neutrons were found to be $0.30 \mathrm{~cm}, 0.81 \mathrm{~cm}$, and $5.8 \mathrm{~cm}$ respectively, in good agreement with expected collision-mean-free-path values for the average-energy neutron in each spectrum.

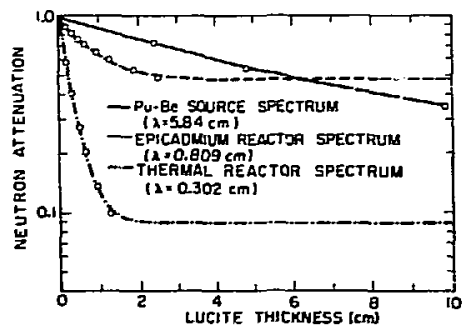

Meyso-4at

Fig. 8. Lucite attenuatiun date for thermal, epicadmium, and fagt neutron energy spectra. The curves are lezst-squares fits of the function $\left.y=C e^{-x}\right)+\{1-C\}$.

Figure 9 shows the results of imaging a test pattern where the standard error in counting statistics per resolution area was approximately equal to the cest-pattern attenuation. The tent pattern was made from 50 mm Mylar giving a measured mean reduction in beam intensity of $2.5 \%$. The standard error in counting statistics per resolution area was $\neq 3 \%$. For Fig. 9c and $d$, at the cost of spatial resolution. the statistics were improved by averaging matrix elements in groups of four.

\section{Discussion}

The performance characteristics of the chamber are in excellent agreement with calculations, with the single exception of the low thermal-neutron detection
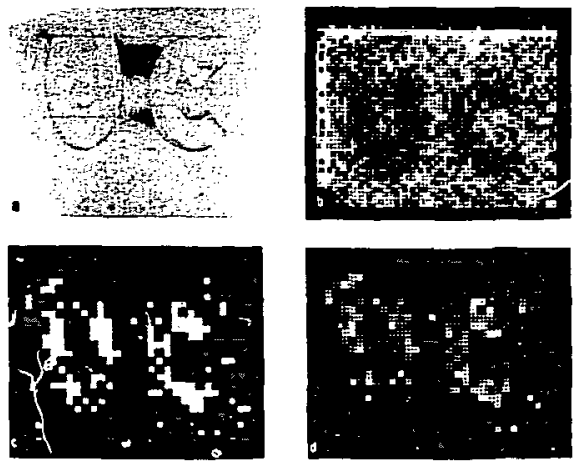

Fig. 9. a) A photograph of the test object-0.002-in. thick Mylar letters on a 0.016-in. -thick aluminum backing.

b) Image produced by normalizing unattenuated intensity to 1.0 and uniformely distributing 32 gray shades between 0.945 and 1.030 .

c) Matrix elements were averaged in groups of four and those lying helow 0.980 were imaged as white.

d) A normal distribution (centered at 0.970 with a $\sigma$ of 0.015 ) was divided into equal-area segments which are shown in 32 shades of gray, with the lightest shede at the center of the distribution.

efficiency (about half thist expected). Considering the data in Table 1 it is quite unlikely to be a detection threshold effect. Two possible sources of this discrepancy are a shorter o-particle range 9 in boron than that used in our calculation and losses due to smell coating irregularities. Efficiency loss factors on the order of $30 \%$ due to this latter effect have been reported,

While fast-neutron detection efficiencies are rather low at present, they might be improved by the application of layered honeycomb converters similar to those recently developed for increasing $\gamma$ Jetection efficiency. 10 By substituting a hydrogenous material for the lead, the effective area for recoil proton emission can be increased.

For biological applications, or for radiation safety, neutron dose is an important consideration. At the present efficiencies achieved for thermal neutron imaging, the neutron radiation dose at the detector is already quite small. For example, for the drill pictures in Fig. 5 the dose ranged from $20 \mu$ rem to 240 prem. The fast-neutron image (Fig. 7) corresponds tgan average detected neutron density of about $17 \mathrm{~mm}^{-2}$ and a detection efficiency of $0.03 \%$ (this reduced efficiency being due to a low high-energy threshold setting made to improve resolucion!. For these conditions the fast neutron dose was about $250 \mathrm{mrem}$. The statistical precision of this image was, however, not warranted by either contrast differtnces or spatial resolution and a comparable quality image could have been obtained at lower fluence. In addition, significant dose reduciion may be achievable by improving fast neutron-converter afficiency. 


\section{Acknowledgments}

The authors would like to express their appreciation to Gerald Stoker for his help in assembling the chamber, and to the UCB reactor staff, particularly Harry Braun and Tek Lim, for providing ug with all but the Pu-Be neutrons.

\section{References}

1. L. Kaufman, V. Perez-Mendez, J. Sperinde, and G. Stoker, Am. J, Röntgenol., Rajium Therapy Nucl. Med. 113, 378 (1971).

2. S. Kaplan, L. Kaufman, V. Perez-Mendez, and K. Val entine, Nucl. Instr. Meth. 106, 397 (1973).

3. K. Valentlne, S. Kaplan, L. Kaufman, and V. Perez-Mendez, IEEE Trans, Nucl. Sci. NS-19, 374 (1972).
4. A. Rindi, V. Perez-Mendez, and R. Wallace, Nucl. Ingtr. Meth. 77, 325 (1970); and R. Grove, I. Ko, B. Leskovar, and V. Perez-Mendez, Nucl. Instr. and Meth. 99, 381 (1972).

5. J. F. Fanni, Tech. Rept, 出AFWL-TR-65-150 (Sept. 1966).

6. K. Rossman, Phys. Med. Biol. 9, 551 (1964).

7. M. R. Hawksworth and M. A. Raoof, J. Phys. E3, $851(1970)$.

8. S. Block and K. F. Petrock, Hazards Control Quarterly Report No. 18, Lawrence Radiation Laboratory Report UCRL-12167 (July-Sept. 1964), p. 19.

9. R. D. Lowde, Rev. Sci. Instr. 21. 835 (1950).

10. C. B. Lim, D. Chu, L. Kaufman, V. PerezMendez, and J. Sperinde, these transactions. 